\title{
An Ex Vivo Evaluation of Cenobamate Administered via Enteral Tubes
}

\author{
Louis Ferrari $^{1} \cdot$ Arkady Nisman $^{1} \cdot$ Augustin Pegan $^{1} \cdot$ Jordan Ursino $^{2}$
}

Published online: 23 April 2020

(c) The Author(s) 2020

Cenobamate Administered via Enteral Tubes

\begin{abstract}
Background Cenobamate is a new, Food and Drug Administration (FDA)-approved oral antiepileptic drug for treatment of focal seizures in adults. This study examined recovery of cenobamate from suspensions administered through ex vivo enteral feeding tubes.

Methods Suspensions containing 100 and $200 \mathrm{mg}$ of cenobamate were prepared (five duplicates for each dose), passed through five vertically standing tubes, and collected into flasks. The flasks containing the suspensions were rinsed with deionized water, and this content was also injected into the tubes and collected in the flasks. Acetonitrile, isopropyl alcohol, and trifluoroacetic acid were added to the flasks, followed by deionized water to a concentration of 500 (100-mg cenobamate) and 400 (200-mg cenobamate) $\mu \mathrm{g} / \mathrm{mL}$. A 3-mL aliquot from each suspension was placed into a 10-mL flask, diluted to volume, and mixed, resulting in final concentrations of 150 and $120 \mu \mathrm{g} / \mathrm{mL}$, respectively. All suspensions were analyzed by high-performance liquid chromatography (LC). The \% LC recovery of cenobamate was calculated for each suspension, and mean $\% \mathrm{LC}$ for duplicates. Results The \% LC recovery of cenobamate from the 100-mg suspensions ranged from 96.2 to $99.1 \%$, with mean \% LC recovery between 96.3 and $98.3 \%$. The \% LC recovery of cenobamate from the 200 -mg suspensions ranged from 97.1 to $102.6 \%$, with mean \% LC recovery between 98.5 and $101.7 \%$.

Conclusion The mean \% LC recovery of cenobamate was within the predetermined acceptable range of $90.0-110.0 \%$, suggesting no adhesion or adsorption of cenobamate to enteral feeding tubes. Delivery of cenobamate suspension via enteral feeding tubes may be a viable route of administration for patients who cannot swallow tablets.
\end{abstract}

\section{Introduction}

Administration of medications via enteral feeding tubes may be necessary in patients who are unable to swallow safely [1]. Patients with dysphagia due to cognitive impairment or physical disability may need to receive medications through enteral feeding tubes [2]. Examples of patients that may require administration of medications by this route include patients with epilepsy, stroke, or dementia [2]. In particular for epilepsy patients, the inability to take an antiepileptic

Prior Presentation/Publication Data from this paper were submitted in abstract form to the American Pharmacists Association 2020 Annual Meeting and Exposition (March 20-23, 2020, National Harbor, Maryland).

Louis Ferrari

lferrari@sklsi.com

1 SK Life Science, Inc., 461 From Road, Fifth Floor, Paramus, NJ 07652, USA

2 Regeneron Pharmaceuticals, Inc., Tarrytown, NY, USA drug may result in loss of seizure control, decreased quality of life, and increased costs due to unscheduled medical visits [2].

Oral liquid formulations of medications in the form of elixirs and suspensions are ideal for administration through enteral feeding tubes [1]. However, certain oral liquid formulations are not appropriate for administration through this route [1]. In addition, oral liquid formulations are not available for many drugs [3]. Alternatively, immediate-release tablets that are sugar- or film-coated may be crushed and mixed with water or hard gelatin capsules may be opened and their contents mixed with water for delivery through an enteral feeding tube [1]. Factors that need to be considered prior to extemporaneous compounding of a liquid formulation from an oral tablet or capsule for administration through an enteral feeding tube include the characteristics of the tube (diameter, composition, tip location) [1, 4], the drug's physicochemical properties (stability, osmolarity, tonicity, adhesion, adsorption, and pharmacokinetics, such as site of absorption) [1-5], and the potential for interactions or 


\section{Key Points}

Mean \% liquid chromatography recovery of cenobamate suspension after passing ex vivo through enteral feeding tubes was within the predetermined acceptable range of 90.0-110.0\%, demonstrating no adhesion or adsorption to the enteral feeding tubes used in this study.

Cenobamate administration via enteral feeding tubes may be a possible option for administration to patients who cannot take anything by mouth. Close monitoring for seizure control and adverse events should be considered when switching the route of administration.

Further studies to confirm the safety and tolerability of cenobamate administration via enteral feeding tubes are warranted.

incompatibilities with enteral nutrition products, diluents, flushes, and other medications that are also being administered in the same tube $[1,2]$.

Cenobamate (YKP3089) is a new, Food and Drug Administration (FDA)-approved oral antiepileptic drug for the treatment of focal (partial-onset) seizures in adults [6]. It has been demonstrated to reduce focal seizures in two phase 2 clinical studies (NCT01397968 and NCT01866111) compared to placebo $[7,8]$. In addition, an interim analysis of an ongoing open-label phase 3 safety study (NCT02535091) demonstrated safety and tolerability of cenobamate over a duration of approximately 6.5 months [9]. Cenobamate is prepared as an oral film-coated tablet that is soluble in water $(1.7 \mathrm{mg} / \mathrm{mL})$. It is recommended to be swallowed whole with liquid and not crushed or chewed [10]. The objective of the present study was to examine the recovery of cenobamate after administration of a suspension prepared from the filmcoated tablets via ex vivo nasogastric and gastrostomy feeding tubes.

\section{Materials and Methods}

Ten suspensions containing $100 \mathrm{mg}$ and ten suspensions containing $200 \mathrm{mg}$ of cenobamate were prepared from the cenobamate 100-mg and 200-mg film-coated tablets that will be marketed, respectively. Duplicate samples of each cenobamate dose strength were passed through five different types of enteral feeding tubes (two cenobamate strength conditions per tube type). A separate tube was used for each preparation.

\subsection{Ex Vivo Administration of Cenobamate via Enteral Tubes}

Cenobamate 100-mg and 200-mg film-coated tablets were placed in 60 and $120 \mathrm{~mL}$ of deionized water in 100- and 200$\mathrm{mL}$ volumetric flasks, respectively. The volumetric flasks were mechanically shaken for $\geq 30$ min until the tablets were completely disintegrated. The resulting cenobamate suspensions were then sonicated for $5 \mathrm{~min}$. The cenobamate suspensions were drawn into $60-\mathrm{mL}$ syringes and injected slowly into five enteral tubes that were vertically standing: tube 1, Port Gientri Salem Sump, Dual Lumen Stomach Tube made of polyvinyl chloride (12 Fr); tube 2, Kangaroo $^{\text {TM }}$ Polyurethane Nasogastric Tube (6.5 Fr neonatal/pediatric nasogastric tube); tube 3, Kangaroo Silicon Gastrostomy Feeding Tube (16 Fr); tube 4, Kangaroo Polyurethane Gastrostomy Feeding Tube (16 Fr); and tube 5, Kangaroo Nasogastric Feeding Tube made of polyurethane (12 Fr). The suspensions of the 100- and 200-mg cenobamate doses were collected into 200- and 500-mL volumetric flasks, respectively, after passing through the tubes. If needed, the tubes could be tapped to retrieve any particles stuck inside for collection into the corresponding volumetric flasks. The tubes did not need to be tapped as there were no particles stuck inside them. The original volumetric flasks containing the 100- and 200-mg cenobamate suspensions were rinsed with $60 \mathrm{~mL}$ and $120 \mathrm{~mL}$ of deionized water, respectively. The rinses were drawn into the same $60-\mathrm{mL}$ syringes used to collect the suspensions, injected into and passed through the five tubes, and collected in the respective 200- and 500-mL volumetric flasks (Fig. 1).

\subsection{Preparation of Cenobamate Filtrates for High-Performance Liquid Chromatography Analysis}

All cenobamate suspensions and their corresponding rinses were collected into 200- and 500-mL volumetric flasks after passing through the five enteral tubes. For the liquid collected from the 100-mg cenobamate suspensions and rinses, acetonitrile $(21.6 \mathrm{~mL})$, isopropyl alcohol $(8.4 \mathrm{~mL})$, and trifluoroacetic acid $(0.1 \mathrm{~mL})$ were added to the $200-\mathrm{mL}$ volumetric flasks. For the liquid collected from the 200-mg cenobamate suspensions, deionized water $(60 \mathrm{~mL})$, acetonitrile $(54 \mathrm{~mL})$, isopropyl alcohol $(21 \mathrm{~mL})$, and trifluoroacetic acid $(0.25 \mathrm{~mL})$ were added to the $500-\mathrm{mL}$ volumetric flasks. All volumetric flasks were then shaken for $\geq 60 \mathrm{~min}$ in a mechanical shaker until all clumps were disintegrated. The volumetric flasks were subsequently sonicated for $5 \mathrm{~min}$. Deionized water was then added to the total volume of the 200- and 500-mL volumetric flasks and mixed well to obtain 


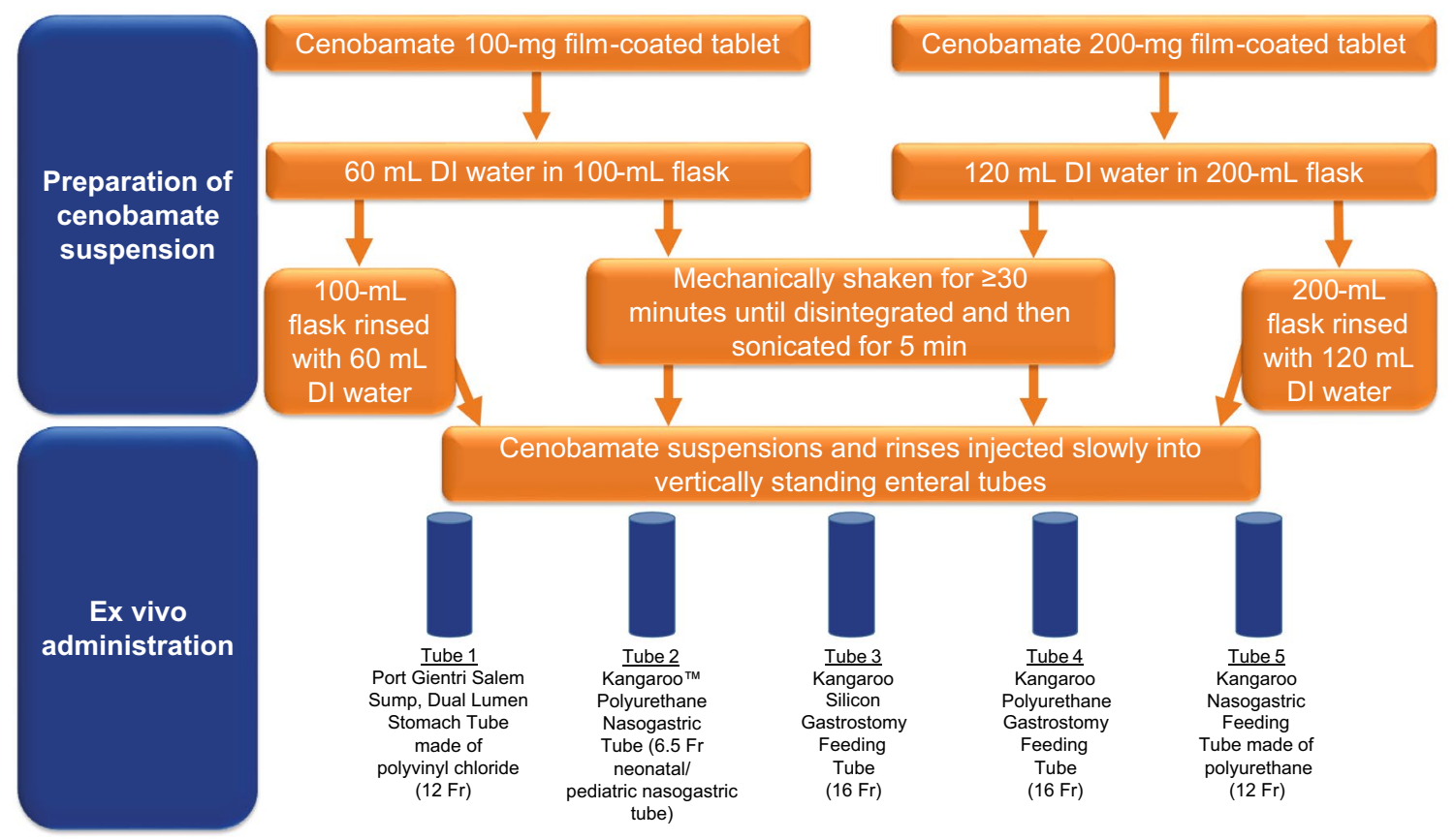

Fig. 1 Ex vivo administration of cenobamate via enteral tube. A total of ten cenobamate suspensions for each dose strength were prepared. Two samples of each cenobamate dose strength were passed through five tubes. DI deionized

a final concentration of 500 and $400 \mu \mathrm{g} / \mathrm{mL}$, respectively. A 3-mL aliquot of each cenobamate suspension was placed into a $10-\mathrm{mL}$ volumetric flask, diluted to a total volume of $10 \mathrm{~mL}$ with diluent and mixed well, resulting in final concentrations of 150 and $120 \mu \mathrm{g} / \mathrm{mL}$ for the 100 - and 200 mg cenobamate strength samples, respectively. All 20 suspensions were then filtered through $0.45-\mu \mathrm{m}$ polytetrafluoroethylene syringe filters, discarding the first $2-3 \mathrm{~mL}$, and the
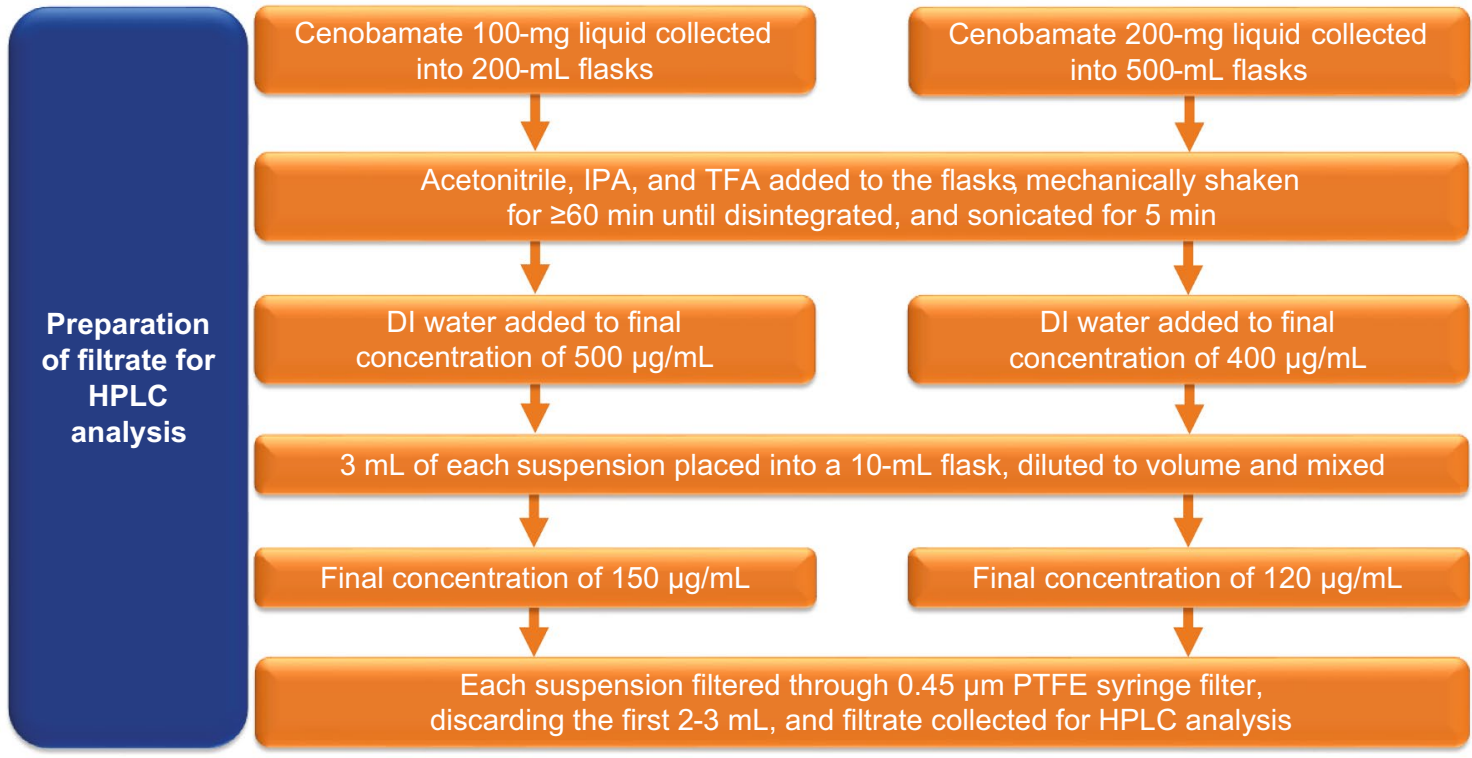

for $\geq 60 \mathrm{~min}$ until disintegrated, and sonicated for $5 \mathrm{~min}$

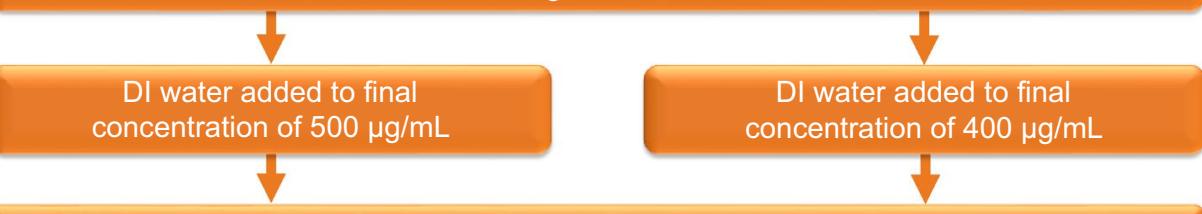

$3 \mathrm{~mL}$ of each suspension placed into a $10-\mathrm{mL}$ flask, diluted to volume and mixed

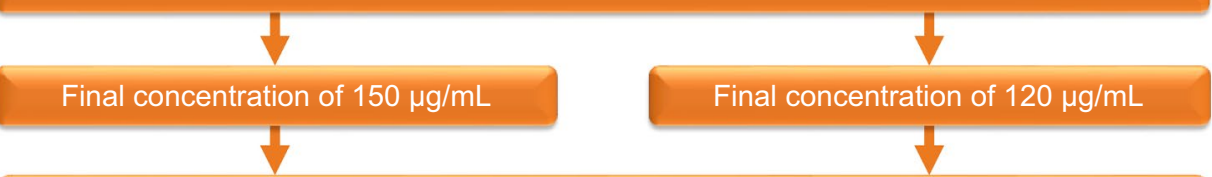

Each suspension filtered through $0.45 \mu \mathrm{m}$ PTFE syringe filter,

discarding the first 2-3 mL, and filtrate collected for HPLC analysis

Fig. 2 Preparation of filtrate for HPLC analysis. DI deionized, HPLC high-performance liquid chromatography, IPA isopropyl alcohol, PTFE polytetrafluoroethylene, TFA trifluoroacetic acid 
filtrates were collected for analysis via high-performance liquid chromatography (HPLC) (Fig. 2).

\subsection{High-Performance Liquid Chromatography Analysis}

The working standard, check standard, and resolution solutions and the 20 cenobamate suspensions (ten duplicates) were analyzed by HPLC. A Phenomenex Gemini C18, $3.0 \mu \mathrm{m}, 150 \mathrm{~mm} \times 4.6 \mathrm{~mm}$ HPLC system was used for the analysis. The sample injection volume was $10 \mu \mathrm{L}$, with a column temperature of $35{ }^{\circ} \mathrm{C}$, and the detection set at UV $220 \mathrm{~nm}$. The mobile phase A was $0.05 \%$ trifluoroacetic acid in water (volume over volume $(\mathrm{v} / \mathrm{v})$ ), and the mobile phase B was $0.04 \%$ trifluoroacetic acid in acetonitrile/isopropyl alcohol $(72: 28, \mathrm{v} / \mathrm{v})$. The flow rate was $1.0 \mathrm{~mL} / \mathrm{min}$, and the run time was $30 \mathrm{~min}$. A standard solution at a concentration of $150 \mu \mathrm{g} / \mathrm{mL}$ was prepared in duplicate from the cenobamate reference standard. The first standard solution was used as the working standard solution, and the second standard solution was used as the check standard solution. A resolution solution was prepared with the cenobamate reference standard and YKP3158, a known impurity, both at a concentration of $150 \mu \mathrm{g} / \mathrm{mL}$. Injection reproducibility was checked with five consecutive injections of the working standard solution and one injection of the check standard solution; a resolution solution was injected to confirm adequate separation between the cenobamate and YKP3158 peaks on the chromatograms. The first injection of the working standard solution was used for the tailing factor and theoretical plates count. System drift was checked with one injection of the working standard solution after injection of every six samples and at the end of the run.

\section{Study Endpoint}

The primary endpoint of the study was the mean $\%$ liquid chromatography (LC) recovery of cenobamate from the duplicate samples passed through the five enteral tubes, for both the 100- and 200-mg cenobamate suspensions. Recovery was considered acceptable if the mean \% LC recovery was within the range of $90.0-110.0 \%$ of the dose. The \% LC recovery was calculated based on the following equation:

$\% \mathrm{LC}=\frac{A_{\text {spl }}}{A_{\text {std }}} \times W_{\text {std }} \times P_{\text {std }} \times \frac{W_{\text {ave }}}{W_{\text {spl }}} \times \frac{\mathrm{DF}_{\text {spl }}}{\mathrm{DF}_{\text {std }}} \times \frac{1}{\mathrm{LC}} \times 100 \%$,

where $A_{\mathrm{spl}}$ is the peak area of cenobamate obtained from the sample preparation, $A_{\text {std }}$ is the average peak area of cenobamate obtained from five injections of working standard preparation, $\mathrm{DF}_{\mathrm{spl}}$ is the dilution factor of the sample preparation (equal to [200 $\times 10] / 3$ for the $100-m g$ strength cenobamate samples and $[500 \times 10] / 3$ for the $200-m g$ strength cenobamate samples), $\mathrm{DF}_{\text {std }}$ is the dilution factor of the working standard preparation, $\mathrm{LC}$ is the label claim of cenobamate per tablet in milligrams, $P_{\text {std }}$ is the potency of the cenobamate reference standard, $W_{\text {ave }}$ is the average weight of the tablet in milligrams, $W_{\text {spl }}$ is the weight of the cenobamate tablet sample powder in milligrams, and $W_{\text {std }}$ is the weight of the cenobamate reference standard.

\section{Results}

Injections checked for both conditions in all five tubes met the system suitability acceptance criteria: injection reproducibility $\leq 2.0 \%$, percentage recovery for check standard solution between 98.0 and $102.0 \%$, resolution between cenobamate and YKP3158 $\geq 2.0$ in the resolution solution, tailing factor from the first injection $\leq 2.0$, number of theoretical plates from the first injection $\geq 4000$, and percentage recovery of system drift injection within $98.0-102.0 \%$.
Table 1 Percentage liquid chromatography recovery of cenobamate after ex vivo administration of the suspension through enteral feeding tubes

\begin{tabular}{llllcc}
\hline $\begin{array}{l}\text { Cenobamate suspension } \\
\text { strength }\end{array}$ & Tube 1 $(\%)$ & Tube 2 (\%) & Tube 3 (\%) & Tube 4 (\%) & Tube 5 (\%) \\
\hline 100-mg tablets & & & & & \\
Sample 1 recovery & 96.7 & 96.2 & 97.8 & 97.0 & 98.3 \\
Sample 2 recovery & 98.4 & 96.3 & 98.8 & 99.1 & 96.5 \\
Mean recovery & 97.5 & 96.3 & 98.3 & 98.0 & 97.4 \\
200-mg tablets & & & & & \\
Sample 1 recovery & 101.3 & 99.9 & 100.9 & 100.4 & 100.4 \\
Sample 2 recovery & 100.0 & 97.1 & 102.6 & 99.7 & 100.4 \\
Mean recovery & 100.6 & 98.5 & 101.7 & 100.0 & 100.4 \\
\hline
\end{tabular}

Tube 1: polyvinyl chloride dual lumen stomach tube (12 Fr); tube 2: polyurethane neonatal/pediatric nasogastric tube (6.5 Fr); tube 3: silicon gastrostomy tube (16 Fr); tube 4: polyurethane gastrostomy tube (16 Fr); tube 5: polyurethane nasogastric tube (12 Fr) 

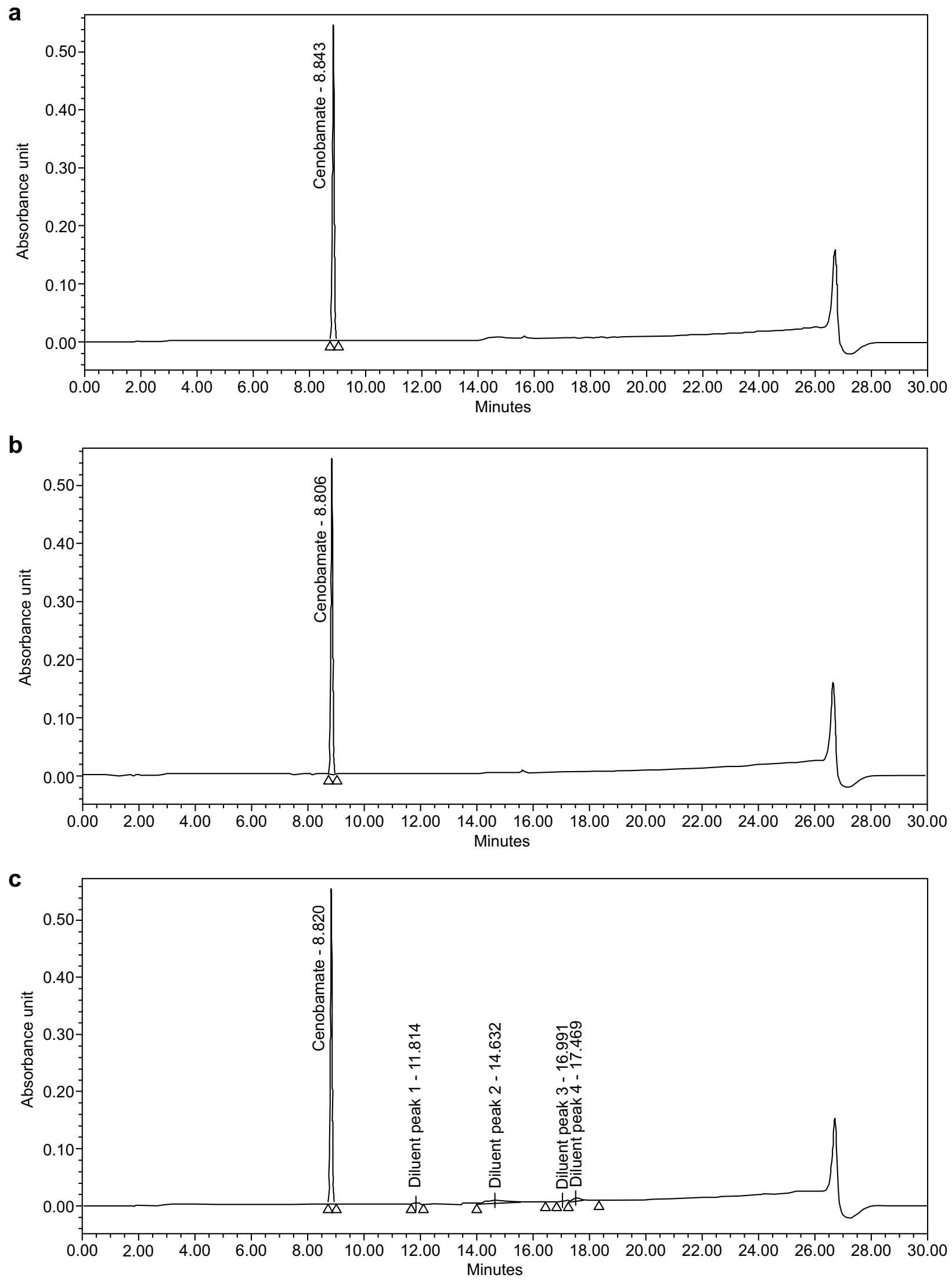

Fig. 3 Chromatograms obtained from 100-mg cenobamate suspension in a tube 1, b tube 2, $\mathbf{c}$ tube 3, d tube 4, and e tube 5 

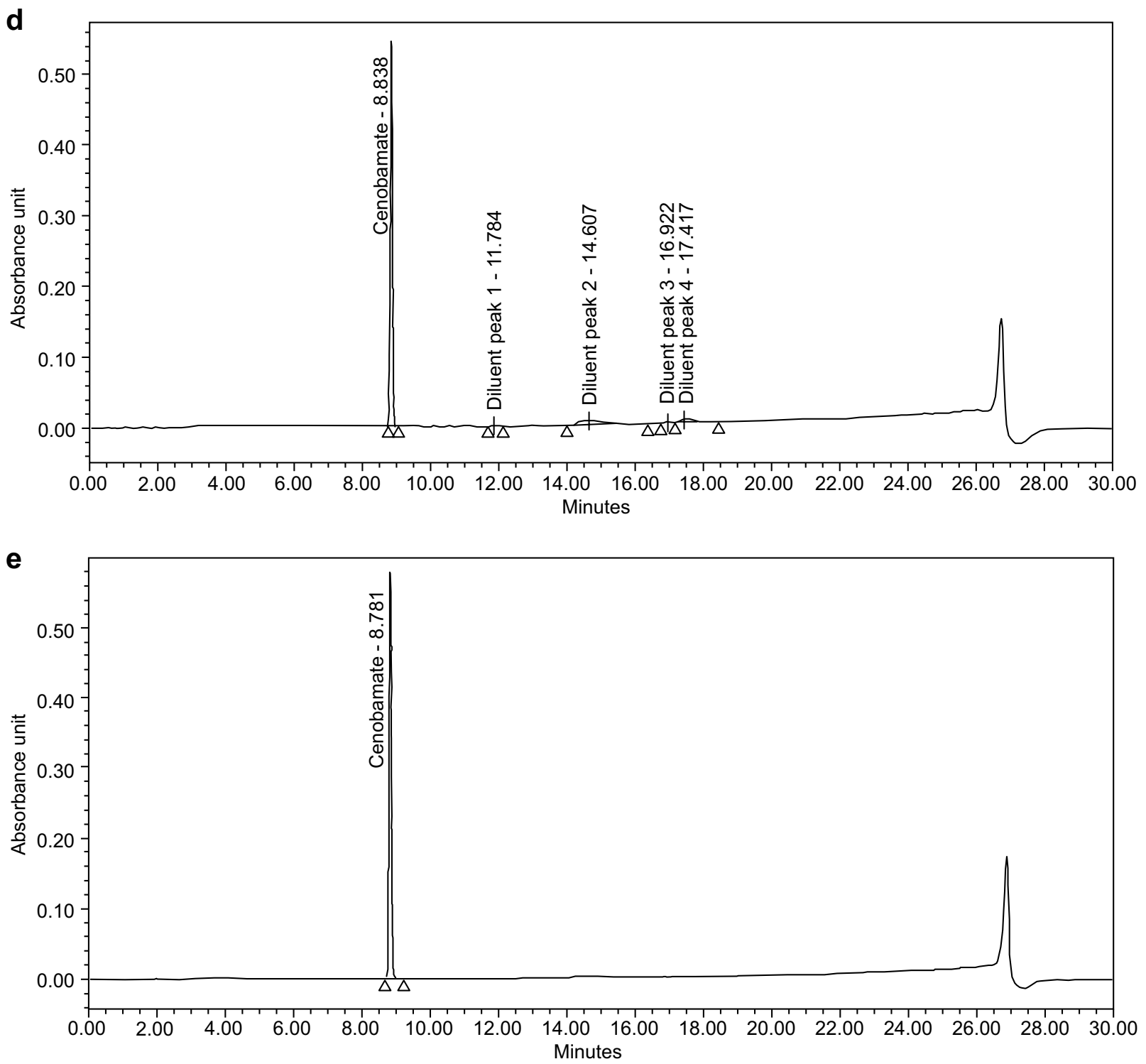

Fig. 3 (continued)

The \% LC recovery of cenobamate from the individual 100-mg suspensions ranged from 96.2 to $99.1 \%$, with the mean \% LC recovery ranging from 96.3 to $98.3 \%$ (Table 1). The $\%$ LC recovery of cenobamate from the individual 200-mg suspensions ranged from 97.1 to $102.6 \%$, with the mean \% LC recovery ranging from 98.5 to $101.7 \%$ (Table 1 ). The retention time of the cenobamate peak was approximately $8.8 \mathrm{~min}$, with an overall chromatographic run time of $30 \mathrm{~min}$. The chromatograms for one sample from each tube for the 100-mg and 200-mg suspensions are depicted in Figs. 3 and 4, respectively.

\section{Discussion}

The mean \% LC recovery of cenobamate was within the specified acceptance criteria of $90.0-110.0 \%$ after ex vivo passage of the suspensions through enteral feeding tubes. There was no significant drug recovery loss with any of the enteral feeding tube sizes and materials used in this study, demonstrating that cenobamate did not adhere or adsorb to the inside of the tubes. The results of this study suggest that delivery of a cenobamate suspension, prepared by dissolving the tablet(s) in water followed by immediate administration, via nasogastric and gastrostomy tubes may be a viable alternative route of administration for patients who are unable to swallow safely. 


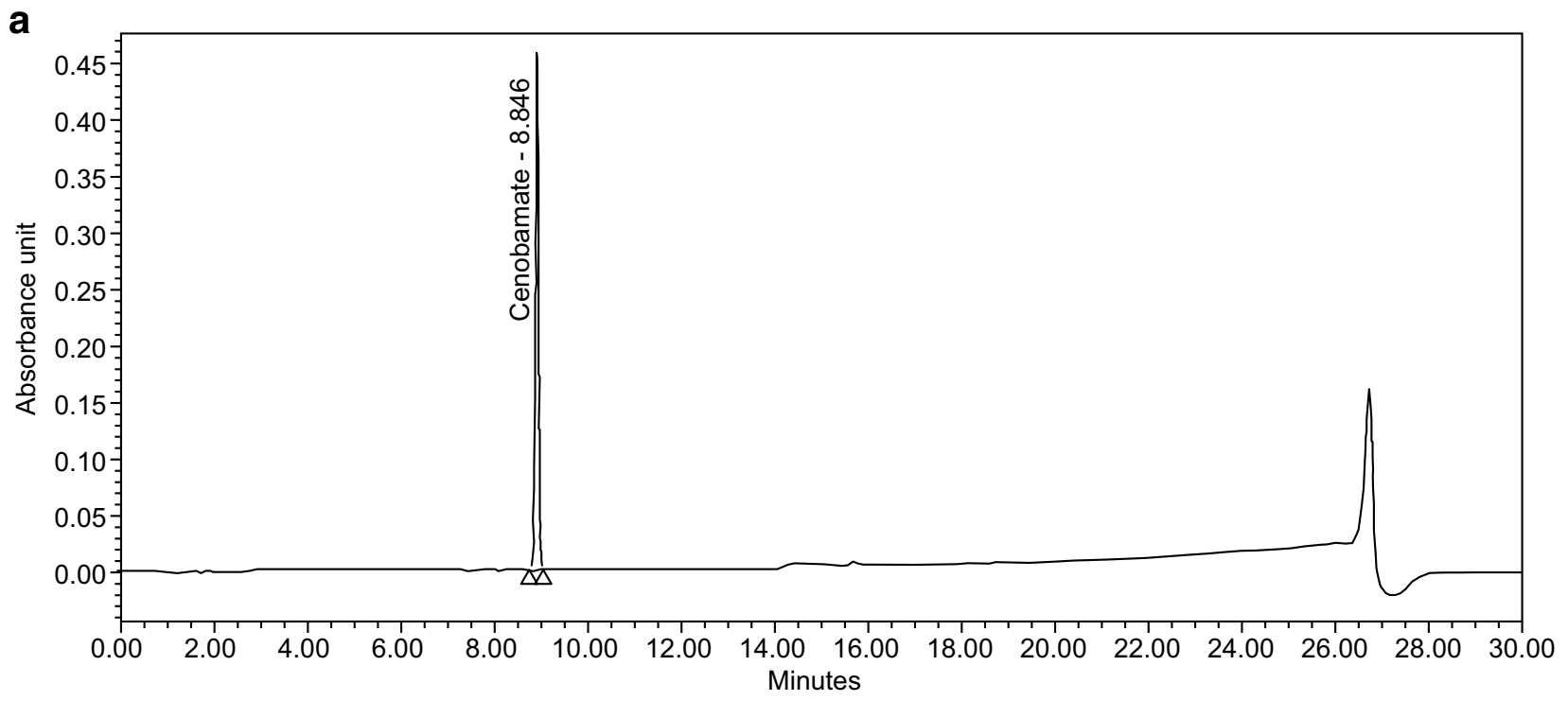

b

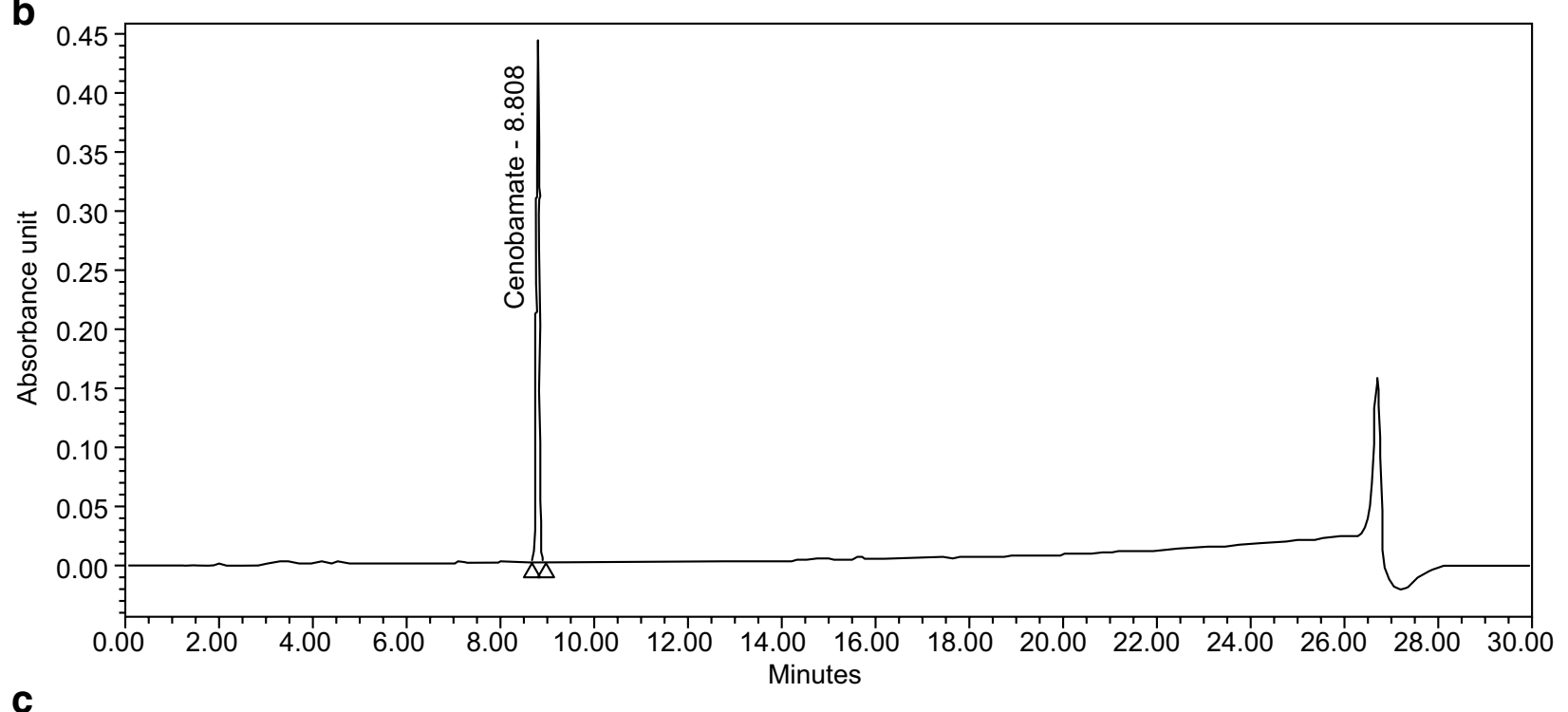

C

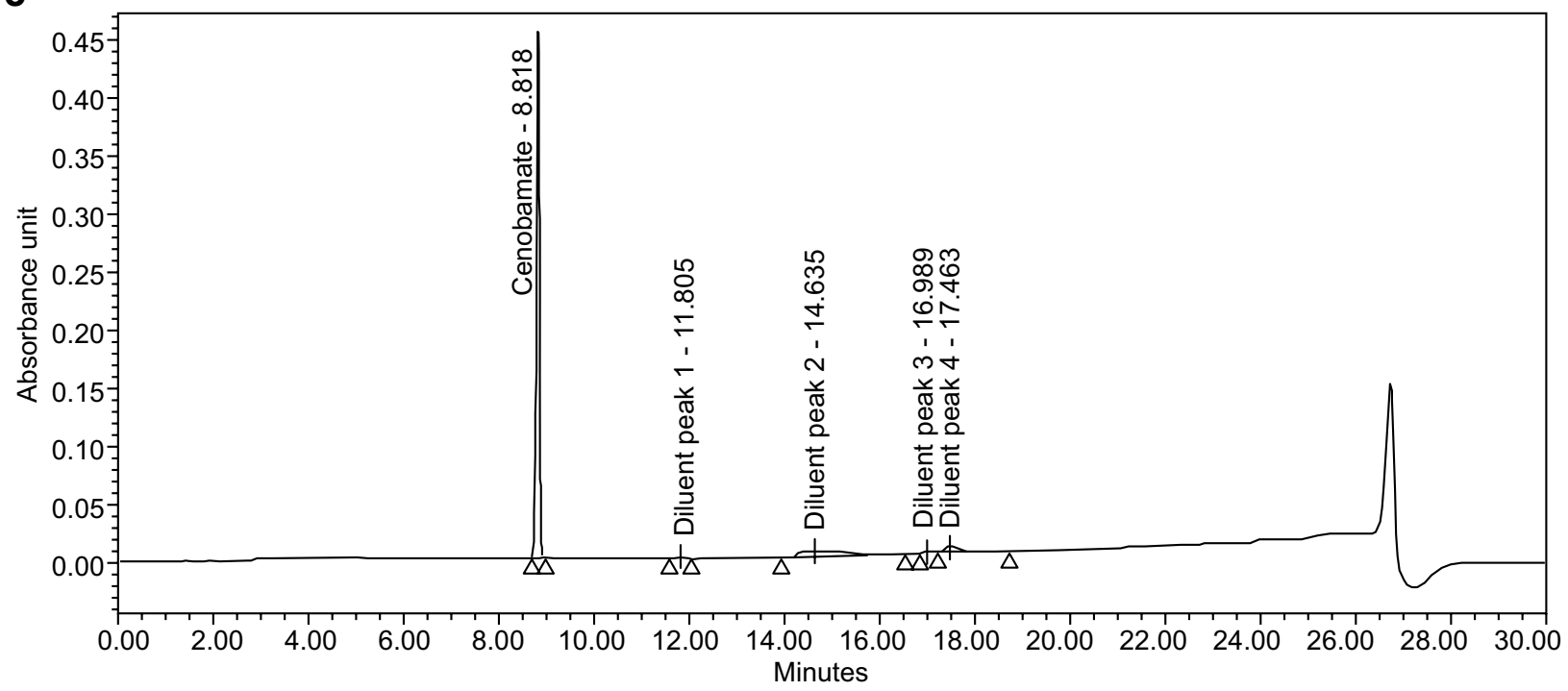

Fig. 4 Chromatograms obtained from 200-mg cenobamate suspensions in a tube 1, b tube 2, c tube 3, d tube 4, and e tube 5 

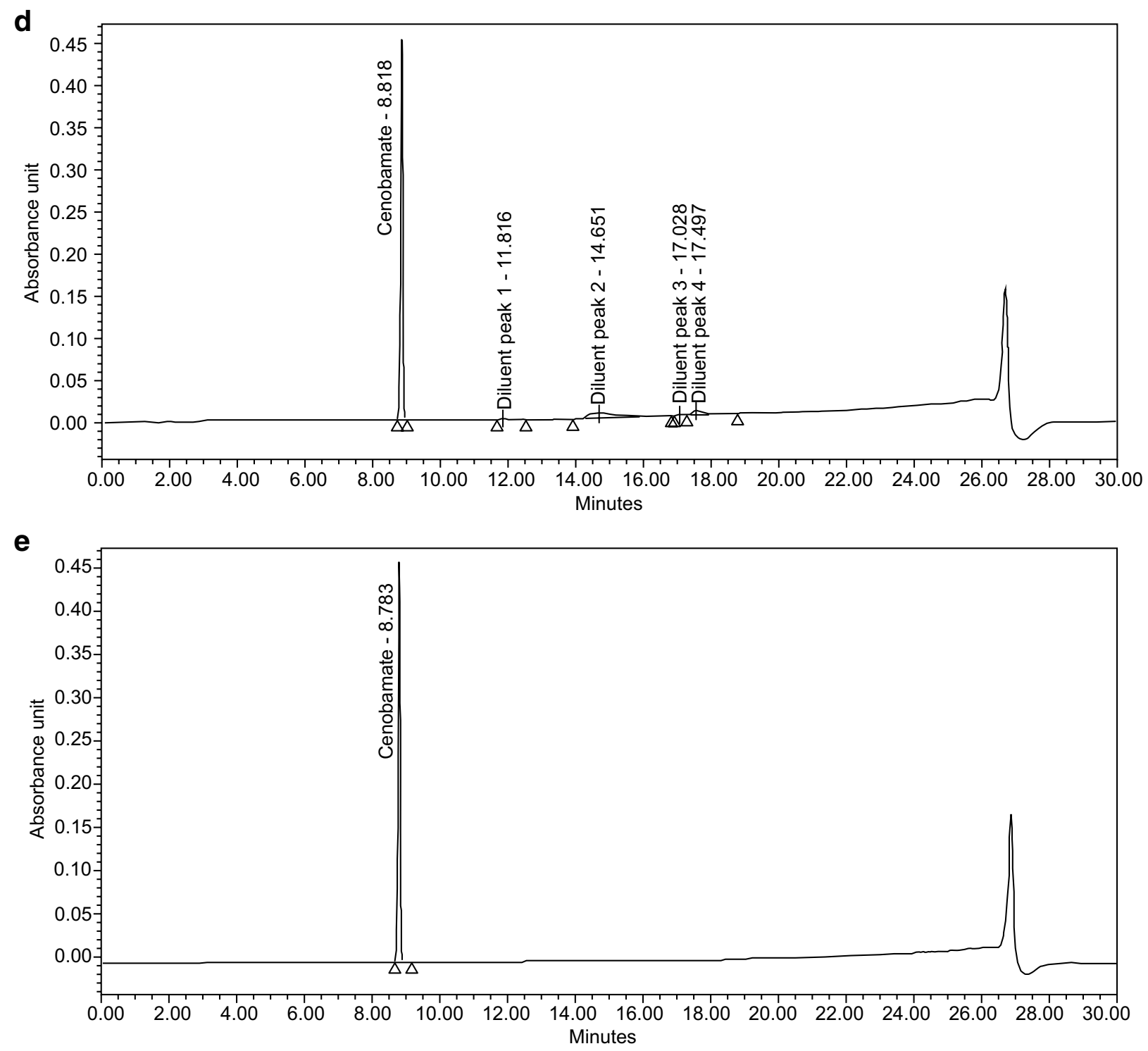

Fig. 4 (continued)

This is the first time that the recovery of cenobamate was assessed after passing the drug suspension through various types of enteral feeding tubes. The study evaluated 6.5-, 12-, and 16-Fr tube sizes and polyvinyl chloride, polyurethane, and silicon tube materials, which represent feeding tubes used in clinical practice. A limitation of this study is that it was performed ex vivo, which may not reflect in vivo pharmacokinetics and pharmacodynamics. Bioavailability and absorption may not be bioequivalent between oral and enteral administration, as feeding tube placement site and adsorption of the drug to the tube can influence these parameters $[1,2,5]$. Serum drug concentrations achieved may differ based on the route of administration, and if available, therapeutic drug monitoring is suggested when changing routes of administration [2].
Possible options for drug delivery in patients who cannot take anything by mouth include enteral feeding tube, transdermal, sublingual/buccal, rectal, intramuscular, subcutaneous, or intravenous administration. The selection of the route is based on the types of formulations available and patient-specific factors, such as mental status, comfort, or intravenous access [1]. Although there are challenges associated with the administration of drugs through enteral feeding tubes (adhesion or adsorption to the inside tube surface, tube occlusions, drug and nutrition incompatibilities, timing in relation to enteral nutrition administration) $[1,4,5]$, the benefits over intravenous administration include decreased cost, convenience, adherence, less risk for infectious complications, should remain as is since less septic complication are associated with enteral feedings which are associated with 
intravenous catheters, and continued use of the gastrointestinal tract, which preserves its function [1, 4]. Development of an aqueous liquid formulation of cenobamate for oral or enteral feeding tube administration is currently ongoing.

\section{Conclusion}

Approximately $100 \%$ of cenobamate was recovered after ex vivo administration of the suspension through enteral feeding tubes. Cenobamate administration via enteral feeding tubes may be a suitable approach to maintain patients on their medication when they cannot take anything by mouth and they have an enteral tube in place. Close monitoring of patients for seizure control and adverse events should be considered when switching between oral and enteral cenobamate administration. Additional studies to verify the safety and tolerability of cenobamate administration via enteral feeding tubes are warranted.

Author Contributions All authors contributed equally, and each author was involved in the study design, data acquisition, or data analysis/ interpretation and in drafting or critically revising the manuscript. All authors reviewed the final manuscript and gave approval for submission.

Data Availability The datasets generated during and/or analyzed during the current study are available from the corresponding author upon reasonable request.

\section{Compliance with Ethical Standards}

Conflict of interest LF: employee of SK Life Science, Inc. AN: employee of SK Life Science, Inc. AP: employee of SK Life Science, Inc. JU: employee of SK Life Science, Inc.

Funding This manuscript was funded, in part, by SK Life Science, Inc. The study was funded by SK Life Science, Inc (Fair Lawn, NJ). The Open Access fee was paid for by SK Life Science, Inc. Professional medical writing assistance and editorial assistance, funded by SK Life Science, Inc, were provided by Dorothy McCoy, PharmD, BCPS BCIDP, of MedVal Scientific Information Services, LLC (Princeton, NJ). This manuscript was prepared according to the International Society for Medical Publication Professionals' "Good Publication Practice for Communicating Company-Sponsored Medical Research: The GPP3 Guidelines."

Open Access This article is licensed under a Creative Commons Attribution-NonCommercial 4.0 International License, which permits any non-commercial use, sharing, adaptation, distribution and reproduction in any medium or format, as long as you give appropriate credit to the original author(s) and the source, provide a link to the Creative Commons licence, and indicate if changes were made. The images or other third party material in this article are included in the article's Creative Commons licence, unless indicated otherwise in a credit line to the material. If material is not included in the article's Creative Commons licence and your intended use is not permitted by statutory regulation or exceeds the permitted use, you will need to obtain permission directly from the copyright holder.To view a copy of this licence, visit http://creativecommons.org/licenses/by-nc/4.0/.

\section{References}

1. Williams NT. Medication administration through enteral feeding tubes. Am J Health Syst Pharm. 2008;65:2347-57.

2. Jory C, et al. Going down the tubes! Impact on seizure control of antiepileptic medication given via percutaneous feeding tubes. Epilepsy Behav. 2017;74:114-8.

3. Beserra MPP, et al. Drugs via enteral feeding tubes in inpatients: dispersion analysis and safe use of dispensers. Nutr Hosp. 2017;34:257-63.

4. Reindel $\mathrm{K}$, et al. In vitro evaluation of eslicarbazepine delivery via enteral feeding tubes. Hosp Pharm. 2017;52:752-60.

5. Moore KT, et al. Rivaroxaban crushed tablet suspension characteristics and relative bioavailability in healthy adults when administered orally or via nasogastric tube. Clin Pharmacol Drug Dev. 2014;3:321-7.

6. US Food and Drug Administration. FDA approves new treatment for adults with partial-onset seizures [press release]. 2019 November 21 [cited 2019 December 6]. https://www.fda.gov/news-event s/press-announcements/fda-approves-new-treatment-adults-parti al-onset-seizures. Accessed Jan 4, 2020.

7. Chung SS, et al. Randomized phase 2 study of adjunctive cenobamate in patients with uncontrolled focal seizures. Neurology. 2019 (in press).

8. Krauss GL, et al. Efficacy and safety of adjunctive cenobamate (YKP3089) in patients with uncontrolled focal seizures: a multicenter, double-blind, randomized, placebo-controlled, doseresponse trial. Lancet Neurol. 2020;19:38-48.

9. Sperling M, Klein P, Kamin M. Safety of cenobamate (YKP3089) as adjunctive treatment for uncontrolled partial seizures in a large, multicenter, open-label study [abstract]. In: American Epilepsy Society Annual Meeting, November 30-December 4, 2018, New Orleans, Abstract 1.303.

10. $\mathrm{XCOPRI}^{\circledR}$ (cenobamate tablets), for oral use, CV [prescribing information]. Paramus: SK Life Science, Inc.; 2020. 